\title{
Supersonic and subsonic stages of dynamic contact between bodies
}

\author{
By M. Adda-Bedia* and Stefan G. Llewellyn Smith ${ }^{\dagger}$ \\ * Laboratoire de Physique Statistique de l'Ecole Normale Supérieure, \\ 24 rue Lhomond, 75231 Paris, France. \\ $\dagger$ Department of Mechanical and Aerospace Engineering, \\ Jacobs School of Engineering, UCSD, \\ 9500 Gilman Drive, La Jolla CA 92093-0411, USA.
}

Using an acoustic model for the full elastic problem, the early times of the twodimensional impact of a disc on a rigid plane, or impact between two identical discs, are analyzed. We examine some aspects of wave propagation during the impact process and we specify stress distributions near the impact region. Unlike the impact of two spheres for which the quasistatic local contact approach of Hertz is well adapted, a complete dynamical approach is necessary for the dynamic contact of two discs. At short times after impact, we show the existence of supersonic effects and we determine the shape of the corresponding stress waves that travel from the impact region through the unstressed body. During the supersonic phase, the contact region grows faster than the speed of sound and the surface outside the contact region is undisturbed. We then solve the transition from supersonic to subsonic regimes and determine the stress distribution near the impact region. Finally, we discuss some physical implications of these results.

Keywords: contact mechanics, stress concentrations, stress waves.

\section{Introduction}

Impact of solid bodies encompasses a wide range of processes. Examples may be found in the fields of tool design, vehicle accidents, game of billiards, granular materials, impact of meteorites, and in many other areas. The concept of impact is differentiated from the case of static, or quasistatic, loading by the nature of its application. Static loading may be regarded as a series of equilibrium states and requires no consideration of accelerating or wave effects. On the other hand, forces created by collisions are exerted and removed in a very short interval of time and initiate stress waves which travel away from the region of contact. However, the complicated process of energy transfer under impact conditions leads to serious difficulties in the mathematical analysis of this type of problems (Goldsmith, 1960; Johnson, 1985).

The foundation for a rational description of impact phenomena was established with the birth of the science of mechanics. The initial concept of rigid-body impact is due to Galileo. Later, Newton furnished the concept of the coefficient of restitution, which has survived essentially unchanged to the present day, though of questionable fundamental significance. An important advance in the field occurred with the treatment of one-dimensional longitudinal and transverse vibrations caused 
by impact on bars (Goldsmith, 1960; Love, 1944). However, this approach was superseded by the Hertz theory of local contact deformations (Landau \& Lifshitz, 1959; Johnson, 1985), which has found wide use in spite of the static elastic nature of its derivation. Using similar approaches, an extensive analysis of the propagation of stresses exceeding the elastic limit, based upon various models of plasticity, has been developed in the last decades (Johnson, 1985).

In the case of a falling sphere on a rigid substrate, the Hertz theory of local contact deformations allows the determination of the duration of impact $t_{H}$ (Landau \& Lifshitz, 1959; Johnson, 1985). It is shown that $t_{H} \propto(c / v)^{1 / 5} R / c$, where $v$ is the initial velocity of the sphere, $R$ its radius and $c$ is a characteristic elastic wave speed of the material. Since in most applications $v \ll c$, the duration of impact is thus much larger than $t_{W} \propto R / c$, the time taken by a wave to travel from the impact region to the upper boundary of the sphere, which is the minimum period of free vibration of the body. Consequently, the quasistatic local contact approach provides reliable results of the stress field for this configuration (Goldsmith, 1960; Johnson, 1985). Nevertheless, at times smaller than $t_{W}$, the propagation of elastic waves from the region of contact into the body cannot be neglected and the stress field in the body exhibits a truly dynamical character (Thompson \& Robinson, 1977). On the contrary, one can show that the Hertz quasistatic approach of impact of an infinite cylinder or of a disc in two dimensions on a rigid plane gives a duration of impact $t_{H} \sim t_{W} \propto R / c$, independently of the initial velocity of the disc (Goldsmith, 1960). Curiously, this two-dimensional impact problem of a disc is similar to the onedimensional case of the impact on bars. Consequently, the quasistatic approximation is not valid for this configuration and one has to deal with a dynamical approach. Finally, independently of the geometry configuration, the dynamics of impact at times smaller than the period of free vibration of the body shows supersonic effects (Thompson \& Robinson, 1977), due to the fact that the contact region spreads more rapidly than the characteristic speeds of elastic waves.

Motivated by the existence of these dynamical processes at the early stages of impact, we examine in this paper some aspects of wave propagation during dynamic contact between elastic solids. During the initial stage of the impact, the spreading velocity of the contact region scales with time as $t^{-1 / 2}$. Therefore, at first contact the spreading velocity exceeds the characteristic wave speed of the body and then decreases with time to become subsonic. Using an elastodynamic formulation of a model linear compressible solid, we analyze the early times of impact between a disc on a rigid plane, or between two identical discs, and we determine stress distributions near the impact region. In particular, we study in detail the transition from supersonic to subsonic regimes. Our dynamical solution of impact is valid for times smaller than the minimum period of free vibration of the body. Note that Thompson \& Robinson (1977) have already drawn attention to the supersonic behavior immediately following the first contact between two spheres. However, they focussed on the supersonic stage of contact between a rigid punch on an elastic body and the transition from supersonic to subsonic regimes was not considered. Moreover, as we shall show below, the dynamics of the contact region of the present problem is different from the case studied in Thompson \& Robinson (1977); Tsai (1971); Bedding \& Willis (1976). Studies of the supersonic and subsonic regimes in the case of impact between a rigid punch and an elastic body have been performed by Kubenko (2004); Borodich \& Gomatam (1998); Zelentsov (2004). The 
case with Coulomb friction has been examined by Brock \& Georgiadis (1994) . We present exact and simple expressions, however, which shed light on the full elastic problem.

The paper is organized as follow. In Section 2, the problem of a falling circular elastic disc impacting a rigid plane, and the corresponding acoustic model problem are presented. In Sections 3 and 4, we determine in detail stress distributions near the impact region for the supersonic and subsonic regimes. Finally, we discuss some physical consequences of our results and their relevance to actual problems.

\section{The dynamic contact problem}

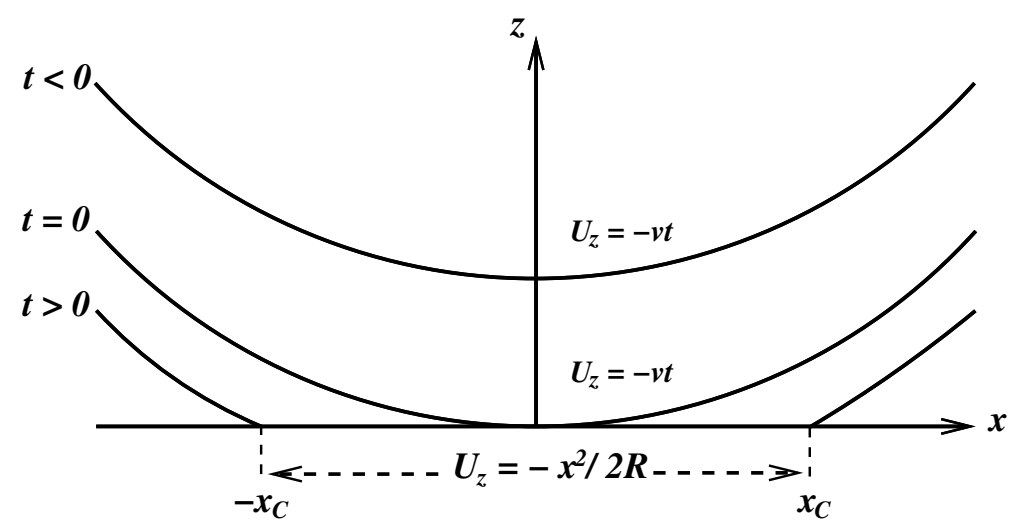

Figure 1. Schematic representation of a falling disc on a rigid substrate. At times $t<0$, the body is falling at constant velocity $v$, so that displacement is given by $U_{z}=-v t$. At time $t=0$, the body touches the rigid plane, and spreads along $z=0$ for $t>0$.

Consider a circular disc of radius $R$ that falls vertically at constant speed $v$, and impacts a rigid plane at time $t=0$ (see Fig. 1 ). We will assume a plane strain deformation of the disc, given by the two-dimensional displacement field $\mathbf{U}(x, z, t)$. Defining $\mathbf{U}(x, z, 0)=0$, the displacement vector at times $t \leq 0$ is simply given by $\mathbf{U}=-v t \mathbf{e}_{z}$, where $\mathbf{e}_{z}$ is a unit vector parallel to the $z$-axis. Let us also define a new displacement vector field $\mathbf{u}$ given by $\mathbf{u}=v t \mathbf{e}_{z}+\mathbf{U}$, which satisfies $\mathbf{u}(x, z, t \leq 0)=0$.

We are interested in the dynamics of impact at times $0<t<t_{W}=2 R / c$, where $t_{W}$ is the time taken by a wave to travel from the impact region to the upper boundary of the disc. During this stage of dynamic contact, the condition on the normal displacement $u_{z}(x, 0, t)$ on the contact region, as defined in Fig.1 by the segment $\left(-x_{C}, x_{C}\right)$, can be derived using the same arguments as for the quasistatic case (Landau \& Lifshitz, 1959):

$$
u_{z}(x, 0, t) \equiv u_{C}(x, t)=v t-\frac{x^{2}}{2 R} \quad \text { for }|x|<x_{C} \text { and } 0<t<t_{W} .
$$

Note that (2.1) is valid when the undeformed configuration of the body in the vicinity of the contact region can be approximated by a parabolic shape. Moreover, the condition (2.1) holds when $0<t<t_{W}$ only, because it assumes that the disc 
is undeformed $(\mathbf{u}=0)$ far from the impact region, which is true until the initial wave radiated from the impact point at $t=0$ has reached the upper boundary of the disc. The wave character of the present problem is similar to the classical one-dimensional vibration problem caused by impact of a bar.

Since the rigid substrate remains undeformed during the impact process, the locations $x= \pm x_{C}$ of the edges of the contact region must satisfy the conditions $u_{C}\left( \pm x_{C}, t\right)=0$, which gives

$$
x_{C}(t)=\sqrt{2 R v t} .
$$

Therefore, the speed of impact spreading in the $x$-direction is given by

$$
\dot{x}_{C}(t) \equiv \frac{d x_{C}}{d t}=\sqrt{\frac{R v}{2 t}}, \quad \text { with } 0<t<t_{W} .
$$

Suppose that the material exhibits one characteristic elastic wave speed $c$. Thus, at short times after the impact the speed of impact spreading is larger than the elastic wave speed of the material $\left(\dot{x}_{C}(t)>c\right)$, which is a signature of supersonic effects. Moreover, the transition from supersonic to subsonic regimes $\left(\dot{x}_{C}(t)<c\right)$ occurs when the dynamic of contact is still governed by (2.1)-(2.3). Effectively, (2.3) shows that the time $t^{*}$ at which the transition supersonic-subsonic regimes occurs is given by

$$
t^{*}=\frac{R v}{2 c^{2}} .
$$

Therefore, within the time interval of interest $\left(0<t<t_{W}\right)$, one has supersonic spreading for $0<t<t^{*}$ and subsonic spreading for $t^{*}<t<t_{W}$.

Let us emphasize that due to the rigidity of the impacted plane, the dynamic spreading of the contact region does not involve the inertia of the disc at times of impact $t<t_{W}$. This behavior is different from the case of impact between a rigid punch and an elastic medium, where the spreading velocity depends of the inertia of the punch (Thompson \& Robinson, 1977; Tsai, 1971; Bedding \& Willis, 1976). In the present configuration, the dynamics of the contact region becomes controlled by the inertia of the disc when $t>t_{W}$ only. Finally, note that the present problem of impact of a disc on a rigid plane is equivalent to impact between two identical discs and the following results are common to both configurations.

\section{(a) The acoustic impact model problem}

In the following, we calculate the elastodynamic response of a disc (or an infinite cylinder) when impacting a rigid plane. We will be mostly interested in the transition from supersonic to subsonic regimes. For this we will use a model solid which exhibits one characteristic wave speed only. The present acoustic model may be connected to elastodynamics by assuming that the shear wave speed of the material vanishes, and thus it will capture the main supersonic-subsonic transition features of the actual elastodynamic problem. We define a scalar field $\varphi$ such that

$$
\mathbf{u}(x, z, t)=\nabla \varphi(x, z, t), \quad p(x, z, t)=-\lambda \nabla^{2} \varphi(x, z, t),
$$

where $\lambda$ is the Lamé elastic constant and $p$ is the pressure field. The present acoustic model is equivalent to supposing that the Lamé shear coefficient vanishes $(\mu=0)$, and that there is only one compressible wave speed given by $c=\sqrt{\lambda / \rho}$, where $\rho$ is 
the material density. Recalling that $t^{*}$ is given by (2.4), we nondimensionalize time by $2 t^{*}$, length by $2 c t^{*}$, displacement by $2 v t^{*}$, pressure by $\lambda v / c$, and $\varphi$ by $4 v c t^{* 2}$. Then the dimensionless transonic time is $t_{T}=\frac{1}{2}$, and the dimensionless period of free vibration of the body becomes $t_{W}=2 c / v$.

For the present acoustic problem, the momentum equations reduce to

$$
\ddot{\varphi}=\nabla^{2} \varphi
$$

with $\varphi(x, z, t<0)=0$. Far from the contact region, the disc is unstressed and thus one has

$$
\varphi(x, z, t)=0 \quad \text { for } \sqrt{x^{2}+z^{2}} \rightarrow \infty .
$$

The condition (2.1) over the region of the boundary $z=0$ in contact with the disc becomes

$$
\varphi_{, z}(x, 0, t)=u_{C}(x, t)=t-x^{2} / 2 \quad \text { for } x^{2}<2 t,
$$

where the subscripted comma denotes partial differentiation. The rest of the boundaries of the disc are traction-free. Thus one has

$$
p(x, 0, t)=-\ddot{\varphi}(x, 0, t)=0 \quad \text { for } x^{2}>2 t .
$$

During the first, supersonic, time interval $\left(0<t<\frac{1}{2}\right)$, the problem has a Neumann boundary condition and can be solved explicitly. A simple expression can be obtained using the Cagniard-de Hoop method. During the second, subsonic time interval $\left(\frac{1}{2}<t<t_{W}\right)$, the problem has mixed boundary conditions with a moving contact line between the two regions, and a more complicated procedure is needed (Poruchikov, 1993).

\section{The supersonic regime}

Acoustic waves in the disc are excited by the imposed displacement (2.8). In the time interval of interest, $t<t_{T} \equiv \frac{1}{2}$, the boundary of the region affected by the impact moves faster than the wave speed and the region $|x|>\sqrt{2 t}$ is not affected. Hence, the unstressed boundaries outside the impact region are not disturbed by the propagation of the acoustic waves from the contact region. Therefore, until the transonic instant an equivalent boundary condition outside the contact area is

$$
\varphi, z(x, 0, t)=0 \quad \text { for } x^{2}>2 t \text { and } t<t_{T} .
$$

Thus, the boundary condition at $z=0$ in the supersonic problem $\left(t<t_{T}\right)$ can be written as

$$
\varphi_{, z} \equiv u_{C}(x, t)=\left(t-x^{2} / 2\right) H\left(t-x^{2} / 2\right),
$$

where $H($.$) is the Heaviside step function.$

Even though the expressions we will obtain only describe the solution until the transonic instant, $t_{T}=\frac{1}{2}$, we calculate them beyond the transonic time, because we will use them in order to solve the subsonic regime. We define the Laplace-Fourier transform by

$$
f^{\mathrm{LF}}(k, s) \equiv \int_{-\infty}^{\infty} \int_{0}^{\infty} f(x, t) \mathrm{e}^{-s(t-\mathrm{i} k x)} \mathrm{d} t \mathrm{~d} x
$$


with inverse transform

$$
f(x, t)=\frac{1}{4 \pi^{2} \mathrm{i}} \int_{-\infty}^{\infty} \int_{a-\mathrm{i} \infty}^{a+\mathrm{i} \infty} s f^{\mathrm{LF}}(k, s) \mathrm{e}^{s(t-\mathrm{i} k x)} \mathrm{d} s \mathrm{~d} k .
$$

Then the Laplace-Fourier transform of the problem takes the form

$$
\begin{aligned}
\varphi_{, z z}^{\mathrm{LF}}(k, z, s) & =s^{2}\left(k^{2}+1\right) \varphi^{\mathrm{LF}}(k, z, s), \\
\varphi_{, z}^{\mathrm{LF}}(k, 0, s) & =u_{C}^{\mathrm{LF}}(k, s)=\sqrt{2 \pi} s^{-5 / 2} \mathrm{e}^{-s k^{2} / 2} .
\end{aligned}
$$

The solution to this problem satisfying (2.7) is found to be

$$
\varphi^{\mathrm{LF}}(k, z, s)=-\frac{u_{C}^{\mathrm{LF}}(k, s)}{s \sqrt{k^{2}+1}} \mathrm{e}^{-s z \sqrt{k^{2}+1}},
$$

We define branch cuts in the $k$-plane extending from $\pm \mathrm{i}$ to $\pm \mathrm{i} \infty$, with $\sqrt{k^{2}+1}$ positive and real for $k$ on the real axis. Hence the transformed pressure is

$$
p^{\mathrm{LF}}(k, z, s)=-s^{2} \varphi^{\mathrm{LF}}(k, z, s)=\sqrt{2 \pi} \frac{s^{-3 / 2}}{\sqrt{k^{2}+1}} \mathrm{e}^{-s k^{2} / 2-s z \sqrt{k^{2}+1}} .
$$

We now consider the Laplace transform of the pressure on the boundary $z=0$, which is given by

$$
p^{\mathrm{L}}(x, 0, s)=\frac{s^{-1 / 2}}{\sqrt{2 \pi}} \int_{-\infty}^{\infty} \frac{\mathrm{e}^{-s\left(k^{2} / 2+\mathrm{i} k x\right)}}{\sqrt{k^{2}+1}} \mathrm{~d} k .
$$

This enables us to write

$$
p^{\mathrm{L}}(x, 0, s)=\pi^{-1 / 2} s^{-1 / 2} g^{\mathrm{L}}(x, s) .
$$

The Cagniard-de Hoop method (e.g. Craster 1996) now relies on defining a new variable $\tau$ so that the integral in (3.9) becomes a Laplace integral. Hence we set

$$
\tau=\frac{1}{2} k^{2}+\mathrm{i} k x
$$

(a) Supersonic region

When $|x|<\sqrt{2 \tau}$, the relation (3.11) may be inverted to give

$$
k(x, \tau)=-\mathrm{i} x \pm \sqrt{2 \tau-x^{2}} .
$$

We now move the Fourier contour to $\operatorname{Im} k=-x$, initially with $|x|<1$ so as not to involve the branch cut. We then change the variable of integration from $k$ to $\tau$. The resulting horizontal contour is described as $t$ takes the values $\frac{1}{2} x^{2}$ to $\infty$ (there are two branches of $k$ corresponding to the two possible signs of the square root: $k_{+}$in the right half-plane and $k_{-}$in the left half-plane). Hence we may write

$$
g^{\mathrm{L}}(x, s)=\frac{1}{\sqrt{2}} \int_{\infty}^{x^{2} / 2} \frac{\mathrm{e}^{-s \tau}}{\sqrt{k_{-}^{2}+1}} \frac{\mathrm{d} k_{-}}{\mathrm{d} \tau} \mathrm{d} \tau+\frac{1}{\sqrt{2}} \int_{x^{2} / 2}^{\infty} \frac{\mathrm{e}^{-s \tau}}{\sqrt{k_{+}^{2}+1}} \frac{\mathrm{d} k_{+}}{\mathrm{d} \tau} \mathrm{d} \tau .
$$


Since $k_{-}^{2}=\left(k_{+}^{2}\right)^{*}$ and

$$
\frac{\mathrm{d} k_{+}}{\mathrm{d} \tau}=-\frac{\mathrm{d} k_{-}}{\mathrm{d} \tau}=\frac{1}{\sqrt{2 \tau-x^{2}}}
$$

we obtain

$$
g^{\mathrm{L}}(x, s)=\int_{0}^{\infty} H\left(\tau-x^{2} / 2\right) \frac{1}{\sqrt{\tau-x^{2} / 2}} \operatorname{Re} \frac{1}{\sqrt{k^{2}+1}} \mathrm{e}^{-s \tau} \mathrm{d} \tau .
$$

The choice of the branch of $k$ is now irrelevant. The expression (3.15) has the form of a Laplace transform, so $\tau$ is in fact the time variable $t$. From now on we replace $\tau$ by $t$, in particular in (3.12). Now (3.15) may readily be inverted to give

$$
g(x, t) \equiv g_{1}(x, t)=H\left(t-x^{2} / 2\right) \frac{1}{\sqrt{t-x^{2} / 2}} \operatorname{Re} \frac{1}{\sqrt{k^{2}(x, t)+1}},
$$

where $k(x, t)$ is defined by (3.12) with $t$ replacing $\tau$. Hence in the supersonic regime, the pressure on the boundary $z=0$ takes the form

$$
p(x, 0, t) \equiv p_{S}(x, t)=\frac{1}{\pi} t^{-1 / 2} * g_{1}(x, t),
$$

where $*$ is the time-convolution operator.

\section{(b) Wave region}

For $|x|>1$, there is still the contribution $g_{1}(x, t)$ from the horizontal contour, but the contour of integration must now also detour around the branch cut, and so there is an extra contribution, $g_{2}(x, t)$, to add which corresponds to $|x|>\sqrt{2 t}$. The appropriate way to write $k(x, t)$ is now

$$
k(x, t)=-\mathrm{i} x+\mathrm{i} \operatorname{sgn} x \sqrt{x^{2}-2 t} .
$$

The new section of the contour corresponds to values of $t$ in the range $\left(t_{a}, \frac{1}{2} x^{2}\right)$, where $t_{a}(x) \equiv|x|-\frac{1}{2}$ is the equation of the ray propagating at the acoustic velocity that passes through the transonic point. We hence obtain

$g_{2}^{\mathrm{L}}(x, s)=\frac{1}{\sqrt{2}} \int_{x^{2} / 2}^{t_{a}} \frac{\mathrm{e}^{-s t}}{(\mathrm{i} \operatorname{sgn} x)\left|k^{2}+1\right|^{1 / 2}} \frac{\mathrm{d} k}{\mathrm{~d} t} \mathrm{~d} t+\frac{1}{\sqrt{2}} \int_{t_{a}}^{x^{2} / 2} \frac{\mathrm{e}^{-s t}}{(-\mathrm{i} \operatorname{sgn} x)\left|k^{2}+1\right|^{1 / 2}} \frac{\mathrm{d} k}{\mathrm{~d} t} \mathrm{~d} t$.

This leads to

$$
g_{2}(x, t)=H\left(x^{2}-1\right) H\left(x^{2} / 2-t\right) H\left(t-t_{a}(x)\right) \frac{\left|k^{2}+1\right|^{-1 / 2}}{\sqrt{x^{2} / 2-t}} .
$$

The pressure hence takes the form

$$
p_{S}(x, t)=\frac{1}{\pi} t^{-1 / 2} *\left[g_{1}(x, t)+g_{2}(x, t)\right] .
$$

Figure 2 shows $p_{S}(x, t)$ for values of time before and after the transonic instant. The curve marked 'subsonic' is not the physical pressure, since the boundary condition (3.2) is not satisfied outside the contact area. The true pressure in the contact region in the subsonic regime will be determined in Section 4 . The convolution integrals (3.17) and (3.21) have integrable singularities at the endpoints $t=x^{2} / 2$ corresponding to the contact line. 


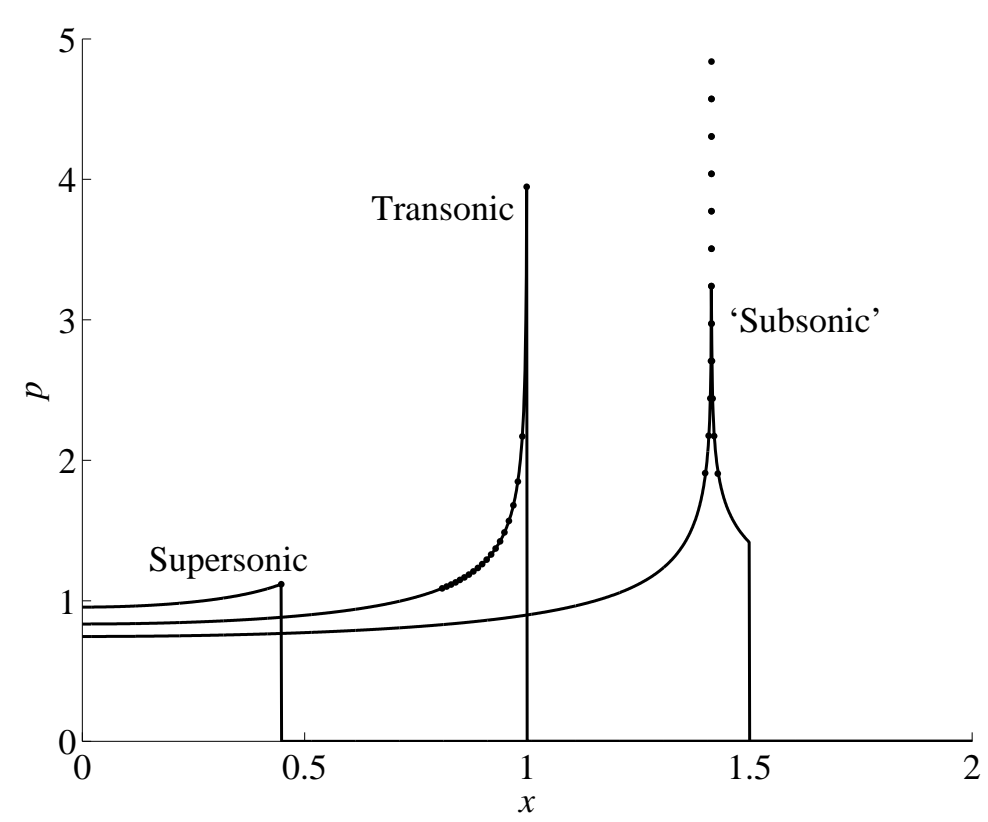

Figure 2. Pressure $p_{S}(x, t)$ at times $t=0.1$ (supersonic), $t=\frac{1}{2}$ (transonic) and $t=1$ (subsonic). The dots correspond to the asymptotic behavior near the contact line given by (3.26) and (3.38).

\section{(c) Asymptotic behaviors}

We now examine in detail the pressure field in the vicinity of the endpoints, $t=x^{2} / 2$, of the contact line. First we consider the $g_{1}$ convolution, which is the only contribution in the supersonic region in which $t<t_{T}$. It can be shown that the pressure given by (3.17) can be written as

$$
p_{S}(x, t)=\frac{2}{\pi} \int_{0}^{\pi / 2} \operatorname{Re} \frac{1}{\sqrt{{k^{\prime}}^{2}+1}} \mathrm{~d} \theta
$$

with

$$
k^{2}+1=1-x^{2}-2 \mathrm{i} x \sqrt{2 t-x^{2}} \sin \theta+\left(2 t-x^{2}\right) \sin ^{2} \theta .
$$

To obtain a uniform limit a new variable is needed. We hence define

$$
a \equiv \frac{2 x \sqrt{2 t-x^{2}}}{1-x^{2}} .
$$

Small values of $a$ correspond to being close to the contact line, but with $x$ not too close to its transonic value of $|x|=1$, while $a \rightarrow \pm \infty$ corresponds to approaching the transonic value of $x$ from the right and left respectively. The integral in (3.23) can then be simplified to

$$
p_{S} \sim \frac{2}{\pi}\left|1-x^{2}\right|^{-1 / 2} \int_{0}^{\pi / 2} \operatorname{Re} \frac{1}{\sqrt{s-\mathrm{i}|a| \sin \theta}} \mathrm{d} \theta,
$$


where $s=\operatorname{sgn}\left(1-x^{2}\right)$. This integral can be expressed in closed form in terms of elliptic integrals, but the resulting formula is not enlightening.

For $x^{2}<1$, i.e. in the supersonic region, we obtain $p_{S}=\left(1-x^{2}\right)^{-1 / 2}=(1-$ $2 t)^{-1 / 2}$ on the contact line. This is the finite value at the right-hand end of the supersonic pressure curve of Figure 2. At the transonic point, which corresponds to $a \rightarrow \pm \infty$, the pressure is singular, with

$$
p_{S} \sim \frac{1}{\pi} \sqrt{\frac{2}{|x|}} K\left(\frac{1}{2}\right)\left(2 t-x^{2}\right)^{-1 / 4} \sim \frac{\sqrt{2}}{\pi} K\left(\frac{1}{2}\right)\left(2 t-x^{2}\right)^{-1 / 4},
$$

where $K$ is the complete elliptic integral of the first kind. This behaviour is indicated by the dots on the transonic curves in Figure 2. For $x^{2}>1$, i.e. in the subsonic region, we have

$$
p_{1} \sim \frac{2|x|}{\pi} \frac{\sqrt{2 t-x^{2}}}{\left(x^{2}-1\right)^{3 / 2}} \sim \frac{2 \sqrt{2 t}}{\pi} \frac{\sqrt{2 t-x^{2}}}{\left(x^{2}-1\right)^{3 / 2}} .
$$

This contribution to the pressure tends to zero on the contact line, and will cancel with a contribution from $g_{2}$ as will be shown. The correction terms to (3.27) are $O\left(a^{3}, a c\right)$ where $c \equiv\left(2 t-x^{2}\right) /\left(1-x^{2}\right)$.

In the subsonic region, the $g_{2}$ convolution needs to be taken into account. There are now two cases depending on whether we are in the contact region with $t>$ $\frac{1}{2} x^{2}>t_{a}(x)$ or in the acoustic region with $\frac{1}{2} x^{2}>t>t_{a}(x)$. In the contact region, the integral can be written as

$$
p_{2}=\frac{1}{\pi} \int_{0}^{\pi / 2}\left[\cos ^{2} \theta+\frac{2 t-x^{2}}{x^{2}-2 t_{a}}\right]^{-1 / 2}\left|k^{\prime 2}+1\right|^{-1 / 2} 2 \sin \theta \mathrm{d} \theta .
$$

The factor $\left|k^{\prime 2}+1\right|^{-1 / 2}$ does not simplify near the contact line, while the first factor becomes non-uniform. We are led to define another new variable

$$
b_{c} \equiv \sqrt{\frac{2 t-x^{2}}{x^{2}-2 t_{a}}},
$$

and small values of $b$ correspond to a region near the contact line but not too close to the transonic point. We now obtain the asymptotic behavior of $p$ for small $b$. We find

$$
p_{2}=\frac{2}{\pi} \frac{1}{\sqrt{x^{2}-1}} \log \frac{2}{b_{c}}+I_{c}-\frac{2|x| \sqrt{x^{2}-2 t_{a}}}{\pi\left(x^{2}-1\right)^{3 / 2}} b_{c}+O\left(b_{c}^{2} \ln b_{c}\right),
$$

where

$$
I_{c} \equiv \frac{2}{\pi} \int_{0}^{\pi / 2} \tan \theta\left[\left|k_{c}^{\prime 2}+1\right|^{-1 / 2}-\left(x^{2}-1\right)^{-1 / 2}\right] \mathrm{d} \theta
$$

and

$$
k_{c}^{\prime}=-\mathrm{i} x+\mathrm{i} \operatorname{sgn} x \sqrt{x^{2}-2 t_{a}} \cos \theta .
$$

There is hence a logarithmic singularity in the pressure field. In the acoustic region, a similar analysis holds with $b_{c}$ replaced by $b_{a}$, where

$$
b_{a} \equiv \sqrt{\frac{x^{2}-2 t}{2\left(t-t_{a}\right)}} .
$$


Then

$$
p_{2}=\frac{2}{\pi} \frac{1}{\sqrt{x^{2}-1}} \log \frac{2}{b_{a}}+I_{a}+O\left(b_{a}^{2} \ln b_{a}\right)
$$

where

$$
I_{c} \equiv \frac{2}{\pi} \int_{0}^{\pi / 2} \tan \theta\left[\left|k_{a}^{\prime 2}+1\right|^{-1 / 2}-\left(x^{2}-1\right)^{-1 / 2}\right] \mathrm{d} \theta
$$

and

$$
k_{a}^{\prime}=-\mathrm{i} x+\mathrm{i} \operatorname{sgn} x \sqrt{2\left(t-t_{a}\right)} \cos \theta .
$$

Close to the contact line, we can write $x^{2}=2 t+O\left(b_{a}^{2}\right)=2 t+O\left(b_{c}^{2}\right)$, so we define a new variable (note that $t>1 / 2$ )

$$
b=\frac{\left|x^{2}-2 t\right|^{1 / 2}}{\sqrt{2 t}-1} .
$$

Then

$$
p_{S}=\frac{2}{\pi} \frac{1}{\sqrt{x^{2}-1}} \log \frac{2}{b}+I_{0}+O\left(b^{2} \ln b, a^{3}, a c\right),
$$

where the contribution from (3.27) has cancelled with the third term in (3.30). Here $I_{0} \equiv \frac{2}{\pi} \int_{0}^{\pi / 2} \tan \theta\left[\left|k_{0}^{\prime 2}+1\right|^{-1 / 2}-\left(x^{2}-1\right)^{-1 / 2}\right] \mathrm{d} \theta, \quad k_{0}=-\mathrm{i} x+\mathrm{i} \operatorname{sgn} x(\sqrt{2 t}-1) \cos \theta$.

The asymptotic behaviours (3.26) and (3.38) are shown in Figure 2.

\section{The subsonic regime}

We have obtained a solution $\varphi_{S}$ satisfying the boundary condition (3.2) over the entire boundary. Now consider the function $\varphi_{P}$ for $t>t_{T}$ that vanishes along with $\dot{\varphi}_{P}$ at $t=t_{T}$ and satisfies the boundary conditions

$$
\ddot{\varphi}_{P}=-\ddot{\varphi}_{S} \quad \text { for } x^{2}>2 t ; \quad \varphi_{P, z}=0 \quad \text { for } x^{2}<2 t .
$$

Then the sum $\varphi=\varphi_{S}+\varphi_{P}$ satisfies the boundary conditions $(2.8,2.9): \varphi_{, z}=u_{C}$ for $x^{2}<2 t$ and $p=-\ddot{\varphi}=0$ for $x^{2}>2 t$, and is continuous in time, as is its timederivative. It is hence the solution to the full physical problem with the correct boundary conditions.

A method of calculating $\varphi_{P}$ is given in Poruchikov $(1993 ; \S 6.4 .3$; see also Slepyan $2002)$. It turns out to be essential to work with the pressure. We write $\ddot{\varphi}=-p=a$ and $\varphi_{, z}=\sigma$. We consider only the right-hand discontinuity and change to the new coordinates $\tilde{x}=x-1, \tilde{t}=t-t_{T}=t-\frac{1}{2}$. Note also that $\tilde{p}_{S}(\tilde{x}, \tilde{t})=p_{S}(x, t)$. The transonic point is at the origin of the new time and space variables. We drop tildes for now.

The contact line is now at the location $l(t)=\sqrt{2 t+1}-1$ (so that $l(0)=0$ and $\left.l^{\prime}(0)=1\right)$. The solution to the problem for $a$ and $\sigma$ is given by

$$
\begin{aligned}
& a_{-}=S_{-} * *\left\{\left[\sigma_{-} * * S_{+}-a_{+} * * P_{-}\right] H[l(t)-x]+C\right\}, \\
& \sigma_{+}=-P_{+} * *\left\{\left[\sigma_{-} * * S_{+}-a_{+} * * P_{-}\right] H[x-l(t)]-C\right\},
\end{aligned}
$$


where the double convolution operator involves both time and space. Variables with subscript $+($ respectively - ) vanish for $x<l(t)$ (respectively $x>l(t)$ ). The boundary conditions give $\sigma_{-}=0$ and $a_{+}=p_{S}$. The functions $S_{-}, P_{-}$and $P_{+}$are given by

$$
S_{-}=-\frac{t_{+}^{-1 / 2}}{\sqrt{\pi}} \delta(t+x), \quad P_{-}=\frac{t_{+}^{-3 / 2}}{2 \sqrt{\pi}} \delta(t+x), \quad P_{+}=-\frac{t_{+}^{-3 / 2}}{2 \sqrt{\pi}} \delta(t-x),
$$

where

$$
f_{+}(t)= \begin{cases}f(t) & \text { for } t>0 \\ 0 & \text { for } t<0\end{cases}
$$

The contribution $C$ corresponds to a homogeneous solution of the problem, which we neglect for now.

A calculation analogous to that of Poruchikov $(1993, \S 6.4 .2)$ gives

$$
p_{-}=\frac{1}{\pi} H[l(t)-x] \sqrt{l\left(t_{0}\right)-x} \int_{l\left(t_{0}\right)}^{x+t} p_{S}(t+x-\xi, \xi) \frac{1}{\sqrt{\xi-l\left(t_{0}\right)}} \frac{\mathrm{d} \xi}{x-\xi},
$$

where $t_{0}$ is the root of the equation

$$
l\left(t_{0}\right)=x+\left(t-t_{0}\right)
$$

(Note that this is really $\tilde{p}_{S}$ above.) This equation gives the value of time, $t_{0}$, at which a ray propagating to the left at the acoustic velocity from the boundary to the point $(x, t)$ intersects the contact line $x=l(t)$. Note that $t>t_{0}$. Figure 3 shows the contact line and acoustic lines in the original $(x, t)$ plane. The vertical lines are the ranges of integration of the convolution integrals that define the subsonic solution for the values of $(x, t)$ indicated by crosses. The solid portion correspond to the $g_{1}$ convolution and the dashed to the $g_{2}$ convolution. The solid and dashdot portions of the diagonal line make up the path of integration for the subsonic solution defined by (4.6) for the supersonic value of $(x, t)$. The dotted portion is the continuation of that straight line to $(x, t)$. Along the dash-dot portion, the integrand (the supersonic potential evaluated beyond the transonic point) vanishes by causality. The solution that we find is valid in the region located in the right half-plane $x>0$ below the crossing of the acoustic ray emitted from the left-hand contact line and the right-hand contact line itself. Beyond that time, we must take into account both transonic points. However we do not need to do this in order to calculate the structure of the solution in the initial phase of the subsonic region. The reason we derived the solution (4.6) to the problem in terms of $p$ is that this produces a solution which has acceptable behaviour in the pressure field. Working with $\varphi$ or $\dot{\varphi}$ gives unacceptable singular terms. These solutions could be eliminated using the homogeneous solutions, but this would be difficult.

We have hence obtained the full solution to the subsonic problem. Figure 4 shows the full solution for times after the transonic instant. Near the contact the pressure is finite, with square root behaviour. We see that as time progresses, the solution adjusts as a disturbance moves to the left from the contact line leading to a cusp. At later times, the solution has a smooth parabolic shape. 


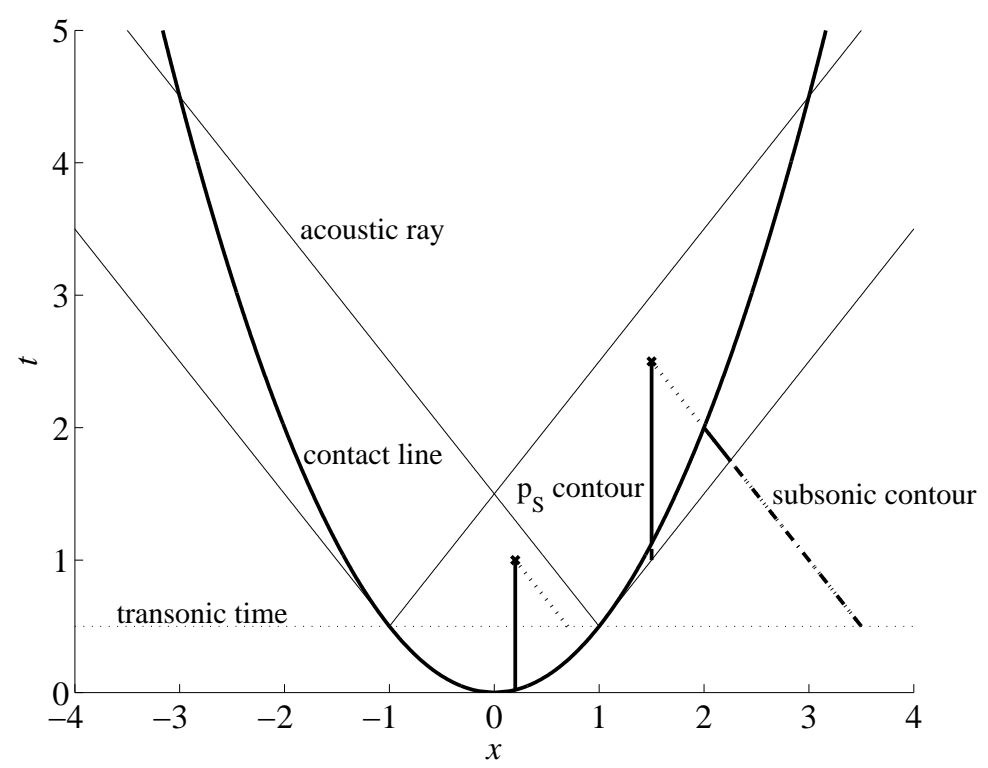

Figure 3. Space-time diagram for the subsonic and supersonic problems. The parabola is the contact line. The thin diagonal lines are the acoustic rays emitted by the two transonic points. The horizontal dotted line separates the subsonic and supersonic regions. The thick lines are explained in the text. The two indicated points are $(1.5,2.5)$ (subsonic) and $(0.2,1)$ (supersonic).

\section{(a) Asymptotics}

We now calculate the behavior of the pressure to the left of the contact line. We return to the original coordinate system, in which $p_{-}$takes the form

$$
p_{-}=\frac{1}{\pi} H[l(t)-x] \sqrt{l\left(t_{0}\right)-x} \int_{l\left(t_{0}\right)}^{x+t-1 / 2} p_{S}(t+x-\xi, \xi) \frac{1}{\sqrt{\xi-l\left(t_{0}\right)}} \frac{\mathrm{d} \xi}{x-\xi} .
$$

The only difference is in the upper limit of the integral. The relation (4.7) takes the same form in the original coordinate system. A convenient variable to investigate the asymptotic behaviour is $\epsilon \equiv l\left(t_{0}\right)-x$. We obtain

$$
p=-\frac{\epsilon^{1 / 2}}{\pi} f_{0}^{t-1 / 2} p_{S}(t-\zeta, l(t)+\zeta) \frac{\mathrm{d} \zeta}{\zeta^{3 / 2}}+O\left(\epsilon, b^{2} \ln b, a^{3}, a c\right),
$$

where the integral is to be understood as a Hadamard principal part integral. The derivation of (4.9) is given in Appendix Appendix A. The coefficient of $\epsilon^{1 / 2}$ is hence a regularized integral of $\varphi_{S}(t, x)$ over the acoustic region (along the bold diagonal line of Figure 3). The variable $\epsilon$ is convenient, but $\eta \equiv l(t)-x$ is more physical. For small $\epsilon$ and $\eta$, one can show that

$$
\epsilon=\frac{\eta}{1-\dot{l}(t)}
$$




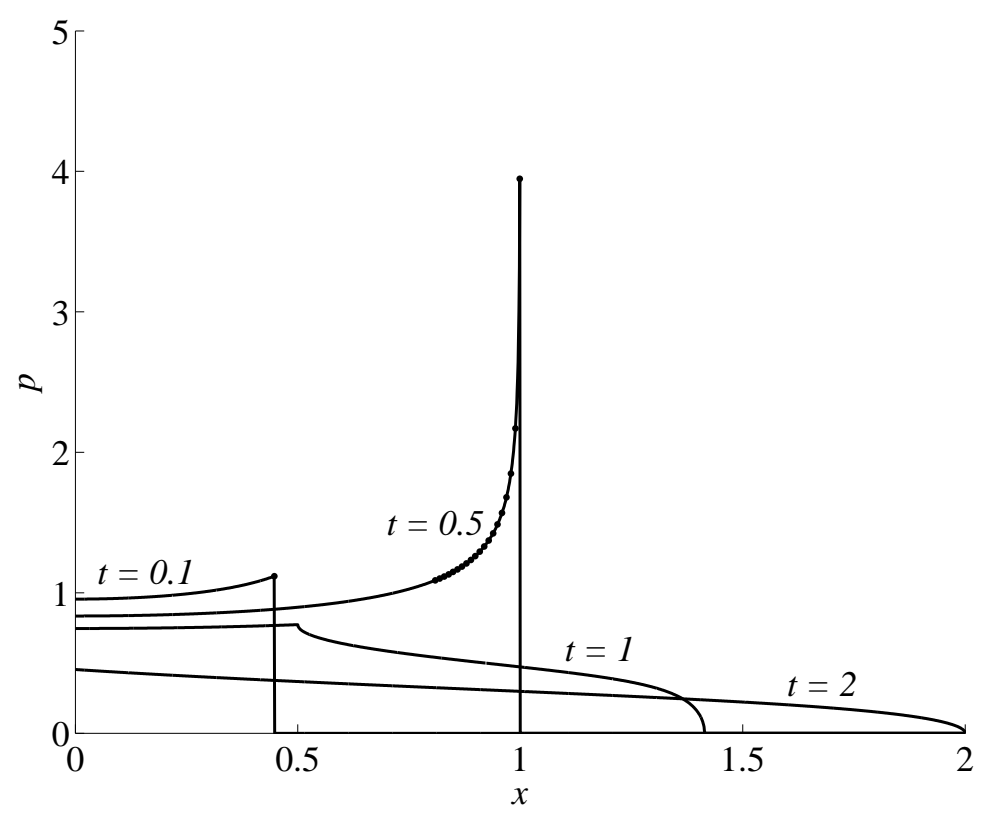

Figure 4. Plots of the full pressure along the contact line at different times of the impact process. The dots correspond to the asymptotic behavior (4.9) near the endpoint $x_{C}=\sqrt{2 t}$

The various error terms in (4.9) then become $b^{2} \ln b \sim \eta \log \eta, a^{3} \sim \eta^{3 / 2}, a c \sim \eta^{3 / 2}$. This leads to

$$
p=-\frac{\eta^{1 / 2}}{\pi(1-i(t))} f_{0}^{t-1 / 2} p_{S}(t-\zeta, l(t)+\zeta) \frac{\mathrm{d} \zeta}{\zeta^{3 / 2}}+O(\eta \ln \eta) .
$$

These asymptotic limits are not uniformly valid close to the transonic point where the various denominators in $a, b$, and $c$ are no longer small.

\section{Discussion}

Some aspects of wave propagation during dynamic contact between solids have been presented. In the framework of a model linear acoustic solid, we have analyzed the earlier times of impact between a disc on a rigid plane, or between two identical discs, and have determined stress distributions near the impact region. In particular, the transition from supersonic to subsonic regimes has been studied in detail. A key feature of the impact of a body on a rigid plane or between two identical discs is that the spreading of the contact region does not involve the inertia of the disc at times of impact $t<t_{W}$. The dynamics of the contact region is determined and its spreading velocity scales with time as $t^{-1 / 2}$. This behavior is different from the case of impact between a rigid punch and an elastic medium, where the spreading velocity depends of the inertia of the punch (Thompson \& Robinson, 1977; Tsai, 1971; Bedding \& Willis, 1976). 
For the present problem, a transonic transition occurs at times $t^{*}=2 R v / c^{2}$ which corresponds to a size $r=2 x_{C}=4 R(v / c)$ of the impacted region, which could be relevant if the impact speed $v$ is large enough. At the endpoints, the stress has been found to be singular as $x^{-1 / 4}$. While this singularity does not induce a finite nonzero energy release rate, it corresponds to large stress concentrations. Moreover, Figure 4 shows that just below $t^{*}$ the stress distribution in the supersonic regime increases rapidly when approaching the endpoints $\pm x_{C}$ and may exceed the elastic limit of the material. On the contrary, in the subsonic regime, the stress tends to zero at these points. Therefore, although a permanent deformation can be produced by exceeding the elastic limit even in the quasistatic loading, the dynamic part of the impact will reinforce this behavior. The impact phenomenon changes in ways which depend upon the mechanical properties of both the projectile and the target. Thus, it can be also conjectured that such a wave propagation singularity might have some effects on the origin of the coefficient of restitution. Moreover, the divergence of the pressure field at the transonic point might contribute to the wear of materials subjected to dynamical contact loadings.

The total force on the over the contact region in the supersonic regime can be calculated quite simply from (3.8). Noting that the pressure in the supersonic regime vanishes outside the contact region, we have

$$
P(t)=\int_{-\infty}^{\infty} p(x, 0, t) \mathrm{d} x=p^{F}(0,0, t)=\frac{2}{\sqrt{\pi}} t^{1 / 2} \propto V(t) S(t) .
$$

This is the same result mentioned by Borodich \& Gomatam (1998) in the supersonic stage of contact. No such simple form appears possible for the subsonic regime.

The extension of the present analysis to an elastic solid is conceptually straightforward. The Cagniard-de Hoop method still works, because the waves are nondispersive, but the space-time diagram becomes more complicated as two different waves are released at the two sonic instants. However, the results can still be expressed in terms of simple convolution integrals and asymptotic limits can be calculated. The subsonic calculations is significantly more complicated however. Once again the method remains the same, but the equivalent result to (4.6) is no longer a single integral, but rather a triple integral. As mentioned previously, we expect the transonic behaviour here to carry through to the full elastic problem.

While the present analysis was restricted to two-dimensional impact problems the results should persist in three dimensions, especially the singular behavior at the transonic point and the stress distribution in the contact region should be similar. Moreover, the general results of the present acoustic model should be relevant to the elastodynamic problem if one restricts oneself to the analysis of the supersonicsubsonic transition regimes. Finally, this study may provide a general framework for dynamic impact problems where the transition from supersonic to subsonic regimes is involved. Effectively, although the fraction of the impact energy which is radiated as waves is generally very small, it may be important in some applications such as seismology (Rosakis, 2002) or the kinetics of granular materials.

We thank Y. Pomeau and W. R. Young for enlightening discussions. We also thank a referee for helpful comments and references. The Laboratoire de Physique Statistique de l'Ecole Normale Supérieure is associated with the CNRS (UMR 8550) and Universities Paris VI and Paris VII. SGLS was supported as a visiting professor from the University 
Paris VII during part of this work and also partly by a Lindemann Trust Fellowship administered by the English Speaking Union while at the Scripps Institution of Oceanography.

\section{Appendix A. Asymptotic behavior of $p_{-}$near the contact point}

We first carry out the change of variable $\xi=l\left(t_{0}\right)+\zeta$ in $(4.8)$, so that to the left of the contact line (where $\epsilon>0$ )

$$
p_{-}=-\frac{\epsilon^{1 / 2}}{\pi} \int_{0}^{t-1 / 2-\epsilon} p_{S}\left(t-\zeta-\epsilon, l\left(t_{0}\right)+\zeta\right) \zeta^{-1 / 2} \frac{\mathrm{d} \zeta}{\zeta+\epsilon} .
$$

Consider the integral

$$
J=\int_{0}^{A} F(\zeta) \zeta^{-1 / 2} \frac{\mathrm{d} \zeta}{\zeta+\epsilon}
$$

where we allow $A$ and $F(\zeta)$ to depend on $\epsilon$. The results of $\S \mathrm{c}$ show that

$$
F(\zeta)=\alpha_{a} \log \beta_{a} \zeta+O(\zeta)
$$

for small $\zeta$, where $\alpha_{a}$ and $\beta_{a}$ are factors depending on the terms in (3.34). Using standard methods, we obtain

$$
J=\epsilon^{-1 / 2} \pi \alpha_{a} \log \beta_{a} \epsilon+I(A)-2 \alpha_{a} A^{-1 / 2}\left(\log \beta_{a} A+2\right)+O\left(\epsilon^{1 / 2}\right),
$$

where the integral $I(A)$ is given by

$$
I(A) \equiv \int_{0}^{A} \frac{F(\zeta)-\alpha_{a} \log \beta_{a} \zeta}{\zeta^{3 / 2}} \mathrm{~d} \zeta
$$

In our case, $A=t-\frac{1}{2}-\epsilon$. From $\S$ c, expanding $A$ in $\epsilon$ gives an $O(\epsilon \ln \epsilon)$ correction to $(A)$. The remaining $O(1)$ part of (A 4$)$ is exactly the definition of the Hadamard finite-part integral

$$
f_{0}^{t-1 / 2} F(\zeta) \frac{\mathrm{d} \zeta}{\zeta^{3 / 2}}=\int_{0}^{t-1 / 2} \frac{F(\zeta)-\alpha_{a} \log \beta_{a} \zeta}{\zeta^{3 / 2}} \mathrm{~d} \zeta-\frac{2 \alpha_{a}}{\sqrt{t-1 / 2}}\left(\log \left[\beta_{a}\left(t-\frac{1}{2}\right)\right]+2\right)
$$

The full asymptotic result for the subsonic pressure in the contact region is hence the combination of (3.27), (3.30), (A 1) and (A 4):

$$
\begin{aligned}
p_{-}= & -\pi \alpha_{a} \log \beta_{a} \epsilon-\frac{\epsilon^{1 / 2}}{\pi} f_{0}^{t-1 / 2} F(\zeta) \frac{\mathrm{d} \zeta}{\zeta^{3 / 2}}+O(\epsilon) \\
+ & \frac{2}{\pi} \frac{1}{\sqrt{x^{2}-1}} \log \frac{2}{b}+I_{c}+O\left(b_{c}^{2} \ln b_{c}, a^{3}, a c\right)
\end{aligned}
$$

(once again the $O\left(b_{c}\right)$ terms have cancelled). We can now replace $b_{a}$ and $b_{c}$ by $b$ in the $\alpha_{a}, \beta_{a}$ and $I_{c}$ terms,making an error of $O\left(b^{2}\right)$. But then the logarithmic and constant terms cancel. The finite-part integral is now to be computed using $\alpha$ and $\beta$, i.e. expressing $p_{S}$ using (3.38) near the contact line.The final result is

$$
p_{-}=-\frac{\epsilon^{1 / 2}}{\pi} f_{0}^{t-1 / 2} p_{S}(t-\zeta, l(t)+\zeta) \frac{\mathrm{d} \zeta}{\zeta^{3 / 2}}+O\left(\epsilon, b^{2} \ln b, a^{3}, a c\right) .
$$




\section{References}

Bedding, R.J. \& Willis, J.R. 1976 High-speed indentation of an elastic half-space by conical or wedge-shaped indentors. J. Elasticity 6, 195-207.

Borodich, F.M. \& Gomatam, J. 1998 An exact solution for the first stage of frictional collision of a die and an elastic half-space. Q. J. Mech. Appl. Math. 51, 563-576.

Brock, L.M. \& Georgiadis, H.G. 1994 Dynamic frictional indentation of an elastic half-plane by a rigid punch. J. Elast. 35, 223-249.

Craster, R.V. 1996 Wavefront expansions for pulse scattering by a surface inhomogeneity. Q. J. Mech. Appl. Math. 49, 657-674.

Goldsmith, W. 1960 Impact. The theory and physical behaviour of colliding solids. Edward Arnold Pub., London.

Johnson, K.L. 1985 Contact mechanics. Cambridge University Press, Cambridge.

Kubenko, V.D 2004 Impact of blunted bodies on a liquid or elastic medium. Int. Appl. Mech. 40, 1185-1225.

Landau, L.D. \& Lifshitz, E.M. 1959 Theory of elasticity. Pergamon Press, London.

Love, A.E.H. 1944 A treatise on the mathematical theory of elasticity. Dover Publications, New York.

Poruchikov, V.B. 1993 Methods of the classical theory of elastodynamics. SpringerVerlag, Berlin.

Rosakis, A.J. 2002 Intersonic shear cracks and fault ruptures. Adv. Phys. 51, 1189-1257.

Slepyan, L.I. 2002. Models and phenomena in fracture mechanics. Springer-Verlag, Berlin.

Thompson, J.C. \& Robinson, A.R. 1977 An exact solution for the superseismic stage of dynamic contact between a punch and an elastic body. J. Appl. Mech. 44, 583-586.

Tsai, Y.M. 1971 Dynamic contact stresses produced by the impact of a axisymmetrical projectile on an elastic half-space. Int. J. Solids Struct. 7, 543-558.

Zelentsov, V.B. 2004 Transient dynamic contact problems of the theory of elasticity with varying width of the contact zone. J. Appl. Maths Mechs 68, 105-118. 